\title{
Expression et régulation de la colère : les effets d'un programme cognitif-comportemental appliqué à des adolescentes hébergées en centre de réadaptation The effects of a cognitive-behavioral program delivered to adolescent females in residential care
}

\author{
Nadine Lanctôt et Annie Lemieux
}

Volume 41, numéro 2, 2012

URI : https://id.erudit.org/iderudit/1061800ar

DOI : https://doi.org/10.7202/1061800ar

Aller au sommaire du numéro

Éditeur(s)

Revue de Psychoéducation

ISSN

1713-1782 (imprimé)

2371-6053 (numérique)

Découvrir la revue

Citer cet article

Lanctôt, N. \& Lemieux, A. (2012). Expression et régulation de la colère : les effets d'un programme cognitif-comportemental appliqué à des adolescentes hébergées en centre de réadaptation. Revue de psychoéducation, 41(2), 209-229. https://doi.org/10.7202/1061800ar
Résumé de l'article

La présente étude évalue les effets d'un programme d'intervention de type cognitif-comportemental sur la régulation et l'expression de la colère chez des adolescentes hébergées en Centres jeunesse. L'évaluation des effets du programme sur la capacité des adolescentes à réguler leur colère et à l'exprimer de façon prosociale a été menée au moyen d'un devis de recherche quasi-expérimental qui permet de comparer 104 adolescentes du groupe expérimental à 78 adolescentes du groupe contrôle sur une période de 18 mois. Les résultats des analyses statistiques indiquent que les adolescentes ayant été hébergées en centre de réadaptation se sont améliorées au plan de la colère ressentie et de la suppression de la colère durant ces 18 mois, qu'elles aient été exposées ou non au programme cognitif-comportemental. Alors qu'aucun changement dans le temps n'a été observé pour le groupe contrôle au regard du tempérament colérique, de l'expression agressive de la colère et de la régulation de la colère, toutefois, toutes ces dimensions s'améliorent dans le temps pour les adolescentes du groupe expérimental. Ces résultats signifient que ces difficultés demeurent constantes et persistantes au fil du temps chez les adolescentes du groupe contrôle, alors qu'elles s'atténuent significativement chez celles qui ont été exposées au programme cognitif-comportemental. Les effets sont congruents avec les objectifs du programme en ce qui concerne la colère et se positionnent donc en faveur de l'application d'un programme de type cognitif-comportemental à des adolescentes hébergées en Centre jeunesse.
Tous droits réservés ( $\odot$ La Corporation de la Revue Canadienne de Psycho-Éducation, 2012
Ce document est protégé par la loi sur le droit d'auteur. L'utilisation des services d’Érudit (y compris la reproduction) est assujettie à sa politique d'utilisation que vous pouvez consulter en ligne. 


\title{
Expression et régulation de la colère : Les effets d'un programme cognitif-comportemental appliqué à des adolescentes hébergées en centre de réadaptation
}

\author{
The effects of a cognitive-behavioral program delivered to \\ adolescent females in residential care
}

\section{N. Lanctôt 1,2,3,4 \\ A. Lemieux ${ }^{1}$}

1. Département de psychoéducation, Université de Sherbrooke. Chaire de recherche du Canada sur la délinquance des adolescents et des adolescentes

2. Cette recherche a été financée par la Stratégie canadienne de prévention du crime, ministère de la Sécurité publique et de la Protection civile du Canada, et par la Chaire de recherche du Canada sur la délinquance des adolescents et des adolescentes.

3. L'auteure désire remercier Émilie Giroux, coordonnatrice de recherche, ainsi que toutes les intervieweuses pour leur apport considérable au bon déroulement de la collecte de données.

4. L'auteure remercie chaleureusement les éducateurs, les éducatrices et les gestionnaires des Centres jeunesse participants pour leur collaboration remarquable au projet de recherche.

\section{Correspondance :}

Nadine Lanctôt

Département de psychoéducation Université de Sherbrooke

150, place Charles-Le-Moyne, bureau 200

Longueuil, Québec J4K 0A8

Tél: 0014504631835 ext. 61833

Fax: 0014504636595

nadine.lanctot@usherbrooke.ca

\section{Résumé}

La présente étude évalue les effets d'un programme d'intervention de type cognitif-comportemental sur la régulation et l'expression de la colère chez des adolescentes hébergées en Centres jeunesse. L'évaluation des effets du programme sur la capacité des adolescentes à réguler leur colère et à l'exprimer de façon prosociale a été menée au moyen d'un devis de recherche quasi-expérimental qui permet de comparer 104 adolescentes du groupe expérimental à 78 adolescentes du groupe contrôle sur une période de 18 mois. Les résultats des analyses statistiques indiquent que les adolescentes ayant été hébergées en centre de réadaptation se sont améliorées au plan de la colère ressentie et de la suppression de la colère durant ces 18 mois, qu'elles aient été exposées ou non au programme cognitif-comportemental. Alors qu'aucun changement dans le temps n'a été observé pour le groupe contrôle au regard du tempérament colérique, de l'expression agressive de la colère et de la régulation de la colère, toutefois, toutes ces dimensions s'améliorent dans le temps pourles adolescentes du groupe expérimental. Ces résultats signifient que ces difficultés demeurent constantes et persistantes au fil du temps chez les adolescentes du groupe contrôle, alors qu'elles s'atténuent significativement chez celles qui ont été exposées au programme cognitif-comportemental. Les effets sont congruents avec les objectifs du programme en ce qui concerne la colère et se positionnent donc en faveur de l'application d'un programme de type cognitifcomportemental à des adolescentes hébergées en Centre jeunesse

Mots-clés : évaluation; intervention; programme de type cognitif-comportemental; adolescentes; colère

\section{Abstract}

This study evaluated the effects of a cognitive-behavioral program delivered to adolescent females placed in residential youth centers on the experience, expression, 


\section{0}

and control of anger. The study used a quasi-experimental design that allowed comparisons between 104 girls in the experimental group and 78 girls in the control group over an 18 months period. Findings revealed that the program had no added value regarding two specific dimensions of anger: felt anger and anger suppression. Indeed, girls from both the experimental and the control group showed similar improvements on these dimensions throughout the 18 months window period. However, adolescents from the experimental group experienced significant decreases over time of difficulties related to choleric temperament, aggressive expression of anger, and anger regulation whereas girls from the control group showed no significant improvements as far as these dimensions were concerned. Since the program does not aim to encourage anger suppression and discourage the externalization of emotions, but rather simply to help girls better regulate and express anger in prosocial ways, our results support the use of cognitive-behavioral programs among adolescent females who engage in problem behaviours.

Key words: evaluation; intervention; cognitive-behavioral program; residential care; adolescent females; anger

\section{Introduction}

II est largement documenté que les conduites agressives causant de sérieux préjudices à autrui sont commises plus souvent par des garçons que par des filles (Loeber \& Hay, 1997; Odgers \& Moretti, 2002; Lauritsen, Heimer, \& Lynch, 2009). Il est cependant de plus en plus reconnu que l'ampleur des conduites agressives commises par des filles ne doit pas être sous-estimée. D'une part, au Canada, les taux de mises en accusation des adolescentes pour des voies de fait ont augmenté de façon fulgurante $(+1,494 \%)$ depuis les trente dernières années, passant de 27/100,000 adolescentes à 429/100,000 adolescentes (Bélanger \& Ouimet, 2010). Bien que ces taux aient légèrement diminué au cours des dernières années, il demeure que de plus en plus d'adolescentes sont amenées devant la justice en raison de délits violents. D'autre part, les avancées sur le plan de la conceptualisation des conduites agressives ont mis en évidence différentes formes d'agression auxquelles se livrent le filles, dont l'agression indirecte (Bjorkgvist \& Niemela, 1992; Crick \& Grotpeter, 1995). Ce type d'agression vise à ternir les relations interpersonnelles d'autrui, et à provoquer le rejet social (Verlaan, Besnard, Déry, Toupin, \& Pauzé, 2009). Lorsque cette forme d'agression est considérée, les taux de prévalence des conduites agressives des filles se comparent largement à ceux des garçons (Crick, Bigbee, \& Howes, 1996). Conséquemment, les filles qui manifestent des conduites agressives, et plus largement celles qui présentent des problèmes sérieux de comportement et de délinquance, sont rapidement devenues une population fortement ciblée par la recherche, ceci afin de mieux comprendre leurs trajectoires développementales, mais aussi, afin de mieux orienter les programmes et les services qui leur sont destinés (Zahn, Day, Mihalic, \& Tichavsky, 2009).

Jusqu'à tout récemment, les réflexions sur le développement de programmes ciblant les problèmes de comportement et de délinquance des filles évoluaient en marge de la littérature scientifique sur les programmes probants. Le développement de programmes spécifiques aux filles était plutôt prôné (Belknap \& 
Holsinger, 1998; Bloom, Owen, Deschesnes \& Rosenbaum, 2002), sans toutefois que les fondements et les effets de ces programmes soient appuyés par des études empiriques (Kempf-Leonard \& Sample, 2000; Lanctôt, 2006). Une nouvelle ligne de pensée se dégage cependant des études les plus récentes dans le domaine de la réadaptation des filles qui présentent des difficultés de comportement. Ces études convergent désormais autour d'une recommandation très claire : implanter des programmes reconnus pour leurs effets probants, tout en adaptant ces programmes et la façon de les dispenser aux besoins particuliers de la clientèle féminine (Barnett, 2012; Hubbard \& Matthews, 2008; Lanctôt, 2010; Zahn et al., 2009). Or, très rares sont les études qui évaluent de façon rigoureuse les effets des programmes d'intervention visant la réduction des difficultés de comportement des filles (Foley, 2008; Hipwell \& Loeber, 2006; Zahn et al., 2009).

L'évaluation de programmes destinés, de façon spécifique ou non, à des filles présentant des difficultés de comportement est un domaine de recherche encore embryonnaire (Hipwell \& Loeber, 2006). Devant l'absence de données probantes sur les programmes d'intervention destinés à cette clientèle, les milieux d'intervention tendent à s'appuyer sur des programmes dont les effets probants ont été rapportés, mais pour la clientèle masculine. À cet effet, plusieurs méta-analyses rapportent que les programmes de type cognitif-comportemental se classent parmi les plus efficaces pour traiter les problèmes de comportement et de délinquance (Landerberger \& Lipsey, 2005; Latimer, Dowden, Morton-Bourgon, Edgar, \& Bania, 2003; Lipsey, 1990; Lösel \& Beelmann, 2006; Sukhodolsky, Kassinove \& Gorman, 2004). Toutefois, les études retenues dans l'ensemble de ces méta-analyses reposent majoritairement, voire entièrement, sur des échantillons masculins. Par exemple, une méta-analyse porte sur les programmes de type cognitif-comportemental qui visent spécifiquement les jeunes présentant des conduites antisociales (McCart, Priester, Davies, \& Azen, 2006). Parmi les 40 études évaluatives recensées, aucune ne porte sur un échantillon composé majoritairement de filles. Quant aux études menées auprès d'échantillons mixtes $(n=16)$, rares sont celles qui rapportent les effets des programmes de façon distincte pour chaque sexe. Devant cette absence de preuves empiriques, l'application de programmes de nature cognitivecomportementale à une clientèle féminine prise en charge en raison de problèmes sérieux de comportement ou de délinquance ne fait pas consensus comme en témoigne Hubbard \& Matthews (2008) lorsqu'ils affirment que : " [t]he application of cognitive-behavioral approaches to female offenders is one of the most contentious areas in programming for female offenders » (p. 238).

D'autres critiques stipulent que les filles et les garçons ont des besoins fondamentalement différents en matière d'intervention. En ce sens, les programmes de nature cognitive-comportementale se centreraient trop sur la colère et les conduites agressives, au détriment d'une intervention visant à atténuer des problèmes plus spécifiques aux filles, comme les troubles intériorisés et les difficultés relationnelles (Cummings \& Leschield, 2002) ainsi que les conditions d'oppression auxquelles elles sont exposées (Kendall \& Pollack, 2003). Or, bien qu'il faille reconnaître que certains besoins en matière d'intervention diffèrent selon le sexe de la clientèle (Belknap \& Holsinger, 1998; Hubbard \& Matthews, 2008; Zahn et al., 2009 ), le besoin d'acquérir des habiletés sur le plan de la régulation de la colère peut difficilement être écarté. À cet effet, une étude menée 


\section{2}

sur l'évaluation des besoins des adolescents et des adolescentes hébergés en centre de réadaptation indique que le besoin de participer à des activités visant la régulation de la colère figure parmi les cibles d'intervention prioritaires, ceci peu importe le sexe de la clientèle (Lanctôt, 2006). Plus précisément, cette étude, menée auprès de 132 adolescentes hébergées en centre de réadaptation, révèle qu'environ $60 \%$ des adolescentes ont reconnu l'importance d'apprendre à mieux régulariser leur colère. Une écrasante majorité d'intervenants (plus de $80 \%$ ) notait aussi l'importance de ces ateliers d'apprentissage pour les adolescentes auprès desquelles ils interviennent.

Par ailleurs, il est bien démontré que les difficultés de comportement, et en particulier les conduites agressives, sont fortement associées à des déficits cognitifs et émotifs en lien avec la colère. Cette association entre les déficits cognitifs et émotifs et les conduites agressives chez les adolescents semble robuste, peu importe qu'elle soit testée auprès d'échantillons de garçons (Cornell, Peterson, \& Richards, 1999; Orobio de Castro, Merk, Koops, Veerman, \& Bosch, 2005), de filles (Marsee \& Frick, 2007; Simmons, 2002), ou mixtes (Fives, Kong, Fueller, \& DiGiuseppe, 2011; Nichols, Graber, Brooks-Gunn, \& Botvin, 2006; Peled \& Moretti, $2007,2010)$. Sur le plan cognitif, la rumination de la colère contribue au maintien et à l'intensification des sentiments négatifs éprouvés (Miller, Pedersen, Earleywine, et Pollock, 2003; Sukhodolsky, Golub \& Cromwell, 2001) et est associée positivement aux conduites agressives (Gobeil, 2008; Repper, 2006, Peled \& Moretti, 2010). La rumination trahit une tendance à centrer ses pensées de manière répétitive, voire intrusive, sur des situations ayant suscité des sentiments négatifs et sur leurs causes et conséquences possibles, sans toutefois s'engager dans une résolution de problèmes (Nolen-Hoeksema, 2004). Les distorsions cognitives génèrent également des réponses agressives, notamment lorsque des intentions hostiles sont attribuées à autrui (Crick \& Dodge, 1994). Sur le plan émotif, la forte réactivité émotionnelle (faible tolérance à la frustration et à la provocation, impulsivité) et la faible régulation des émotions (efforts et moyens déployés pour apaiser ses émotions négatives et éviter une réaction explosive) contribuent à la manifestation de conduites agressives (Cole, Michel, \& Teti, 1994; Conway, 2005; Fabes et al., 1999; Keenan, 2000; Silk, Steinberg, \& Morris, 2003). La suppression de la colère tend aussi à être associée à des problèmes de comportement, particulièrement chez les filles à risque (Cole, Zahn-Waxler, Fox, Usher, \& Welsh, 1996). Ainsi, que la colère soit exprimée d'une façon trop réactive ou qu'elle soit sur-contrôlée en étant supprimée, ces mécanismes dénotent des difficultés à parvenir à réguler sainement et de façon prosociale la colère ressentie (Conway, 2005).

Les programmes de nature cognitive-comportementale ciblent ces déficits liés à la régulation des émotions et leurs effets positifs sur les problèmes reliés à la colère et les conduites agressives des enfants et des adolescents sont bien documentés (voir notamment les méta-analyses de Beck \& Fernandez, 1998; Blake \& Hamrin, 2007; Sukhodolsky, Kassinove \& Gorman, 2004). Plus spécifiquement, ces programmes visent une restructuration cognitive et l'acquisition d'habiletés sociales de façon à ce que les individus puissent gérer leurs émotions de façon prosociale et déployer des stratégies prosociales pour résoudre leurs problèmes. Ces études considérent toutefois peu la clientèle féminine et se limitent surtout aux effets à court terme des programmes. II devient donc pertinent d'évaluer les effets 
que peut avoir un tel programme sur la capacité des adolescentes à réguler leur colère, ceci sur une période de 18 mois. C'est précisément l'objectif du présent article.

\section{Le programme cognitif-comportemental appliqué aux adolescentes héber- gées en centre de réadaptation}

Le programme cognitif-comportemental évalué dans le cadre de la présente étude réfère précisément au programme défini par Le Blanc, Dionne, Proulx, Grégoire, \& Trudeau-LeBlanc (1998). Ce programme s'adresse aux jeunes en difficulté, sans spécificité au regard du sexe. Il est implanté, entre autres, au sein de neuf unités d'hébergement pour adolescentes du Centre jeunesse de MontréalInstitut universitaire (CJM-IU). Une coordonnatrice clinique s'assurait de l'intégrité du programme et offrait de la formation continue aux éducateurs et éducatrices qui appliquaient le programme. L'objectif du programme est de réduire la fréquence des cognitions et des comportements antisociaux, ceci en favorisant l'augmentation de la fréquence des cognitions et des comportements prosociaux. Le programme mise sur une restructuration cognitive des individus et sur l'apprentissage de comportements prosociaux alternatifs. À travers diverses activités du programme, les individus sont amenés à identifier leurs distorsions cognitives, à les remettre en question et à adopter des conduites prosociales alternatives qui seront renforcées par les intervenants. Le programme place aussi les individus dans un contexte d'apprentissage dans le but d'améliorer leurs habiletés sociales et personnelles. En ce qui concerne la colère, le programme vise notamment à ce que les adolescentes apprennent à reconnaître leurs émotions de colère et à identifier les perceptions et les déclencheurs associés à cette émotion. Le programme vise aussi à ce que les adolescentes acquièrent des moyens d'autorégulation afin d'être en mesure d'exprimer leur colère de façon socialement acceptable. II s'agit donc de comprendre que, bien que la colère soit inévitable dans les interactions sociales, et parfois même légitime, cette émotion peut être exprimée, mais à travers un certain filtre rendant la réaction moins explosive et plus adaptée socialement.

Au sein des unités d'hébergement pour adolescentes du CJM-IU, ce programme s'opérationnalise à travers des activités individuelles et de groupe, telles que définies dans le manuel "Intervenir autrement : Un modèle différentiel pour les adolescents en difficulté » (Le Blanc et al.,1998). Ce manuel sert de guide à l'implantation du programme. Voici ce qui est prescrit par le programme. D'une part, le volet individuel comporte trois outils cliniques : une analyse synthèse évaluative (analyse fonctionnelle et analyse des excès-déficits), les auto-observations et le contrat comportemental. D'abord, l'éducateur analyse les composantes problématiques de l'adolescente et propose des solutions de rechange prosociales à adopter. Cette évaluation permet d'identifier les conditions qui précèdent la manifestation d'une conduite problématique et les conséquences qui en découlent (les renforcements vs les punitions). Ensuite, des auto-observations sont effectuées au moins quatre fois par semaine par l'adolescente. Pour chaque auto-observation, l'adolescente rapporte une situation problématique ou positive qui s'est produite ainsi que les pensées, les émotions, les sensations, les comportements et les conséquences qui l'ont suivie. Ce matériel est utilisé, de façon non-structurée, lors de rencontres individuelles avec l'éducateur. L'éducateur définit enfin un contrat 
comportemental avec l'adolescente. Ce contrat, qui doit être renouvelé aux trois mois, spécifie des moments de vie précis au cours desquels l'adolescente doit remplacer un comportement problématique donné par un comportement prosocial spécifique. Le choix du comportement ciblé repose sur l'analyse fonctionnelle réalisée au préalable. Misant sur le renforcement des conduites et des attitudes prosociales, les conséquences liées au respect (renforcements) ou à la transgression (punitions) du contrat sont précisées. Quant au volet collectif, il consiste en quatre ateliers d'apprentissage : l'acquisition d'habiletés de communication, la régulation de la colère, la gestion du stress et la résolution de problèmes. Chaque atelier d'apprentissage est animé par un duo d'éducateurs au sein même des unités d'hébergement et comporte 15 séances hebdomadaires d'une durée d'environ 75 minutes. Les ateliers sont offerts à environ six adolescentes à la fois. Chaque adolescente doit participer à au moins deux séances par semaine, de sorte qu'à la fin de son placement, elle ait complété les quatre ateliers. En conformité avec le principe de l'imitation, les éléments d'apprentissage sont présentés sous forme de modelage au cours de chaque séance. Des exemples significatifs, tirés du vécu des adolescentes, sont soulevés et un jeu de rôle est ensuite effectué pour mettre en application l'habileté enseignée.

\section{La fidélité d'implantation du programme : un fossé entre la théorie et la pratique}

L'implantation du programmes dans un contexte réel de pratique pose toutefois son lot de défis, tel que le démontre l'évaluation de la fidélité d'implantation (Lanctôt, 2012). Le degré de participation au programme cognitif-comportemental a été évalué systématiquement pour toutes les adolescentes du groupe expérimental. Pour chaque adolescente, un éducateur attitré devait remplir une grille qui indique : (1) si une analyse des excès-déficits a été complétée, (2) si un contrat comportemental est actif, (3) le degré d'application de ce contrat, (4) le nombre d'auto-observations faites par semaine, (5) si une rétroaction a été faite sur les auto-observations et (6) la nature et le nombre d'activités de groupe auxquelles l'adolescente a participé. Cette grille permet ainsi de quantifier de façon précise et individualisée le degré de participation au programme, ceci pour toutes les semaines du placement.

Les résultats révèlent que, bien que toutes les adolescentes du groupe expérimental étaient exposées au programme cognitif-comportemental, toutes n'y ont pas été participé à la même intensité au cours de leur placement. Le degré de participation au programme est loin d'être optimal. D'abord, une proportion considérable d'adolescentes n'a jamais participé à l'une ou l'autre des activités de groupe. Plus précisément, $20 \%$ des adolescentes n'ont participé à aucune séance de l'activité visant l'acquisition d'habiletés de communication. Cette proportion de non-participation est de $42 \%$ pour l'activité sur la gestion du stress, 33\% pour l'activité de la régulation de la colère et $22 \%$ pour la résolution de problèmes. De plus, seule une minorité d'adolescentes a complété une portion significative des activités de groupe, soit au moins dix des quinze séances prévues (33\% pour les habiletés de communication, $9 \%$ pour la gestion du stress, $19 \%$ pour la régulation de la colère et $36 \%$ pour la résolution de problèmes). Au total, en additionnant le nombre de séances complétées à travers les quatre ateliers d'apprentissage, les adolescentes ont participé en moyenne à 23.92 séances (écart-type $=10,32$ ), ce qui se situe environ à mi-chemin de ce qui est prévu par le programme (15 séances 
pour chacune des quatre activités de groupe). II importe toutefois de reconnaître que des éléments d'apprentissage pouvaient être abordés avec les adolescentes dans le contexte du vécu partagé (par exemple, pendant le repas ou lors d'une activité sportive). Cependant, une telle exposition au programme ne pouvait pas être quantifiée.

En ce qui concerne les activités individuelles, le degré de participation varie grandement d'une activité à l'autre. Plus précisément, l'analyse des excèsdéficits a été complétée à la fréquence prévue par les éducateurs pour $78 \%$ des adolescentes. L'application du contrat cognitif-comportemental n'atteint pas les critères attendus, soit parce que aucun contrat n'a été défini (13\% des adolescentes) ou parce que leur fréquence d'application était insuffisante ( $50 \%$ des adolescentes). Seul le tiers des adolescentes (37\%) ont eu un contrat actif pendant la majorité de leur placement. Enfin, les auto-observations étaient complétées au moins quatre fois par semaine par une large majorité d'adolescentes (80\%). Toutefois, ce matériel était peu souvent analysé systématiquement avec l'éducateur. Cette analyse a été conduite à la fréquence prévue pour seulement 19\% des adolescentes.

Ces résultats relèvent les difficultés réelles que pose l'application du programme cognitif-comportemental. Une implantation intègre de ce programme semble très difficile, autant au regard des activités de groupe que des activités individuelles. L'évaluation des effets du programme devra donc être interprétée à la lumière de cette réalité bien imparfaite.

\section{Méthodologie}

\section{Le devis de recherche}

L'étude évaluative a été menée au moyen d'un devis quasi-expérimental. Un groupe contrôle et un groupe expérimental étaient comparés, afin que les effets spécifiques du programme cognitif-comportemental soient isolés. Le groupe expérimental était formé d'adolescentes qui séjournaient dans des unités d'hébergement du CJM-IU, là où le programme décrit plus haut était implanté. Le groupe contrôle était composé d'adolescentes hébergées dans un autre Centre jeunesse. Ces adolescentes étaient encadrées au même titre que les adolescentes du groupe expérimental par une équipe d'éducateurs et d'éducatrices, sans toutefois être exposées à un programme d'intervention de nature cognitive-comportementale. Les adolescentes des deux groupes ont complété des questionnaires à leur admission à l'unité d'hébergement (pré-test), de même que trois mois (post-test), six mois (suivi 1), 12 mois (suivi 2) et 18 mois après (suivi 3 ).

\section{La procédure}

De janvier 2008 à octobre 2009, chaque adolescente admise dans l'une des unités d'hébergement ciblées par la présente étude était invitée à participer à la recherche. La coordonnatrice de recherche contactait alors chacune des adolescentes quelques jours après leur admission pour leur expliquer la nature du projet de recherche et les modalités de participation. La participation à la recherche était volontaire et le consentement d'un parent était requis pour les adolescentes 


\section{6}

de 14 ans et moins. Une fois qu'elle avait consenti à participer à la recherche, l'adolescente était rencontrée individuellement et dans un contexte privé par une intervieweuse qui lui soumettait le questionnaire à remplir (pré-test). L'équipe d'intervieweuses était constituée d'étudiantes universitaires formées sur les questions d'éthique et sur les techniques d'entrevues. Le questionnaire, d'environ 90 minutes, comportait diverses sections que l'adolescente complétait seule, ou avec l'assistance de l'intervieweuse. Une fois l'entrevue complétée, l'intervieweuse raccompagnait l'adolescente dans son unité d'hébergement. Trois mois (post-test), 6 mois (suivi 1), 12 mois (suivi 2) et 18 mois (suivi 3) plus tard, chaque adolescente était recontactée par la coordonnatrice de recherche afin de remplir de nouveau un questionnaire. La même démarche que celle qui prévalait pour le pré-test était alors répétée. Du pré-test au post-test (3 mois plus tard), toutes les adolescentes de l'échantillon étaient placées dans une unité résidentielle, bien qu'elles pouvaient passer du temps dans la communauté pour faciliter leur réinsertion sociale. À compter du suivi 1 , soit 6 mois après l'admission, le placement de certaines adolescentes était échu. Dans ce cas, la passation des questionnaires s'effectuait chez les adolescentes.

\section{La stratégie d'échantillonnage}

Afin d'être admissibles à l'étude évaluative, les adolescentes devaient être soumises à un placement dans l'une des unités d'hébergement ciblées pour une période d'au moins trois mois, soit jusqu'au post-test. L'objectif de la recherche était d'évaluer les effets d'un programme d'intervention, comparativement à un placement " classique " en centre de réadaptation. Une exposition significative à ce programme, ou encore un placement d'une durée significative, était donc nécessaire. Or, lors de l'admission des adolescentes dans les unités d'hébergement, la durée de leur placement n'était pas toujours déterminée (certaines adolescentes pouvaient s'y retrouver pour pallier une situation d'urgence ou être réorientées vers d'autres ressources après une période d'évaluation). De façon à maximiser la taille de l'échantillon, toutes les adolescentes admises dans les unités d'hébergement et pouvant possiblement être rencontrées dans les jours suivants ont été invitées à participer au pré-test. Selon ce critère, le nombre d'adolescentes ciblées pour constituer les groupes expérimental et contrôle se chiffrait respectivement à 189 et à 184. Le taux de participation s'est élevé à $83 \%(n=157)$ dans le premier groupe et à $88 \%(n=162)$ dans le second, ce qui signifie que l'échantillon de départ était représentatif des adolescentes hébergées au sein des deux centres de réadaptation. La non-participation était liée à des refus $(n=33)$, à des difficultés logistiques comme des conflits d'horaire $(n=16)$ ou à la trop grande vulnérabilité de la santé mentale des adolescentes $(n=5)$. Parmi les adolescentes ayant complété le pré-test, seules celles qui étaient toujours dans les unités d'hébergement lors du post-test, soit trois mois plus tard, ont été retenues aux fins d'évaluation. Au total, $108(34 \%)$ adolescentes avaient déjà quitté le centre de réadaptation et 211 $(66 \%)$ y étaient toujours hébergées. Le nombre d'adolescentes pouvant figurer dans le devis évaluatif se chiffrait ainsi à 211 (126 dans le groupe expérimental et 92 dans le groupe contrôle). Des analyses ont été conduites afin de s'assurer que l'attrition n'altérait pas la représentativité de notre échantillon. Outre le fait que les adolescentes ayant quitté le centre de réadaptation étaient plus âgées que celles qui y résidaient toujours (15,75 ans vs. 15,18 ans au pré-test), les adolescentes 
retenues dans le devis évaluatif ne se distinguaient pas de celles qui y ont été exclues au regard de l'ampleur des difficultés de comportement rapportées au prétest, ni des différentes dimensions de la colère évaluées dans le cadre du présent article.

Parmi les 211 adolescentes admissibles au devis évaluatif, 182 (86\%) ont accepté de poursuivre leur participation à la recherche et de compléter le questionnaire du post-test, soit $104(87 \%)$ dans le groupe expérimental et 78 (85\%) dans le groupe contrôle. C'est spécifiquement sur cet échantillon que portait l'évaluation du programme. L'âge moyen à l'admission était de 15.12 ans (é.-t. = $1,32)$. La majorité des adolescentes $(60 \%)$ rapportait être d'origine ethnique blanche, alors que $22 \%$ affirmaient être d'origine noire, $8 \%$ d'origine latino-américaine, $4 \%$ d'origine arabe et $6 \%$ d'origine autre. La majorité des adolescentes était placée en raison de troubles de comportement sérieux (43\%), de négligence parentale (25\%) ou de maltraitance (20\%). Le tableau 1 détaille la taille de l'échantillon final et les taux de rétention pour chaque temps de mesure. Au sein du groupe expérimental, $79 \%$ $(n=82)$ des adolescentes ont complété le questionnaire lors du suivi $1,75 \%(n=78)$ au suivi 2 , et $92 \%(n=96)$ au suivi 3 . Ces taux de rétention étaient respectivement de $69 \%(n=54), 62 \%(n=48)$ et $78 \%(n=61)$ pour le groupe contrôle.

Tableau 1. Taille de l'échantillon et taux de rétention selon le groupe d'appartenance (groupe expérimental ou groupe contrôle) et selon le temps de mesure

\begin{tabular}{lccccc}
\hline & $\begin{array}{c}\text { Pré-test } \\
\text { (Admission) }\end{array}$ & $\begin{array}{c}\text { Post-test } \\
(3 \text { mois })\end{array}$ & $\begin{array}{c}\text { Suivi } 1 \\
(6 \text { mois })\end{array}$ & $\begin{array}{c}\text { Suivi 2 } \\
(12 \text { mois })\end{array}$ & $\begin{array}{c}\text { Suivi 3 } \\
(18 \text { mois })\end{array}$ \\
\cline { 2 - 6 } Groupe & $\mathrm{n}=104$ & $\mathrm{n}=104$ & $\mathrm{n}=82$ & $\mathrm{n}=78$ & $\mathrm{n}=96$ \\
Expérimental & $(100 \%)$ & $(100 \%)$ & $(79 \%)$ & $(75 \%)$ & $(92 \%)$ \\
Groupe Contrôle & $\mathrm{n}=78$ & $\mathrm{n}=78$ & $\mathrm{n}=54$ & $\mathrm{n}=48$ & $\mathrm{n}=61$ \\
& $(100 \%)$ & $(100 \%)$ & $(69 \%)$ & $(62 \%)$ & $(78 \%)$ \\
\hline
\end{tabular}

Afin de bien situer l'évaluation, il importe de rappeler que la durée des placements variait d'une adolescente à l'autre. Bien que toutes étaient soumises à un placement entre le pré-test et le post-test (donc pour une durée minimale de trois mois), le placement pouvait être échu à compter du suivi 1 (6 mois après l'admission) et les adolescentes pouvaient se voir offrir de plus en plus d'occasions de sortir du centre de réadaptation à des fins de réinsertion sociale. La grande majorité des adolescentes (88\%) ayant participé au devis évaluatif était encore placée en centre de réadaptation lors de l'évaluation menée 6 mois après leur admission. La proportion chute considérablement lors des évaluations conduites 12 mois (46\%) et 18 mois (28\%) après l'admission. Ces variations sont prises en compte (contrôlées statistiquement) dans l'évaluation des effets du programme. 
Enfin, bien que la répartition des adolescentes au sein des groupes n'était pas aléatoire, les groupes étaient largement équivalents au moment de l'admission au centre de réadaptation. L'âge moyen des adolescentes du groupe contrôle était cependant plus élevé que celui des adolescentes du groupe expérimental $(15,7$ ans vs. 14,7 ans; $p<0,001)$. Des analyses effectuées sur l'ensemble des données du pré-test indiquaient toutefois que, lorsque l'effet de cette différence d'âge était contrôlé statistiquement, les adolescentes de deux groupes se comparaient sur les dimensions émotives (ex: symptômes intériorisés, colère ressentie), cognitives (ex: rumination, stratégies d'adaptation), relationnelles (ex: styles interpersonnels, relations avec les parents) et comportementales (ex: propension aux vols et à la violence) (Ayotte, Lanctôt, Lemieux, \& Giroux, 2009). Quelques différences isolées apparaissaient néanmoins entre les groupes lors du pré-test, notamment en regard d'une des variables ciblées dans le présent article. Lors du pré-test, les adolescentes du groupe expérimental rapportaient de moins bonnes capacités à réguler leur colère comparativement aux adolescentes du groupe contrôle $(F=$ $4,28 ; p<0,05)$.

\section{Les mesures}

Les adolescentes ont rempli un questionnaire portant sur différents thèmes. Aux fins du présent article, les effets du programme cognitif-comportemental ont été évalués spécifiquement en regard des différentes dimensions qui se rattachaient à la colère, ceci à l'aide d'une version traduite du State-Trait Anger Expression Inventory-2 (STAXI-2, Spielberger, 1999). La traduction a été réalisée par l'équipe de recherche. La version originale de ce questionnaire, validée auprès d'adolescents et de patients psychiatriques, était composé de 57 items qui évaluaient de façon objective l'intensité de la prédisposition à la colère, la colère ressentie, de même la suppression, l'expression et la régulation de cette colère (Spielberger, 1999). Les items étaient évalués sur une échelle Likert en quatre points où $1=$ pas du tout, $2=$ un peu, $3=$ assez et $4=$ beaucoup. Pour les fins de la présente étude, des échelles spécifiques ont été sélectionnées en raison de leur rapprochement avec les objectifs du programme évalué et de façon à éviter la présence de corrélations trop élevées entre certaines échelles. Les dimensions évaluées sont décrites cidessous:

Colère ressentie (alpha de Cronbach $=0,96$ ): Cette échelle comporte 15 items qui mesurent l'intensité des sentiments de colère ressentie. Un score élevé à cette échelle signale que l'adolescente rapporte vivre de forts sentiments de colère (ex. : je me sens enragée) et qu'elle a envie de les exprimer par des gestes d'agression verbale (ex. : j'ai envie de crier après quelqu'un) et physique (ex. : j'ai envie de frapper quelqu'un).

Suppression de la colère (alpha de Cronbach $=0,58$ ): Cette échelle inclut huit items qui évaluent à quelle fréquence des sentiments de colère sont supprimés (ex. : quand je suis en colère, je garde tout en dedans de moi; quand je suis en colère, je sens à l'intérieur de moi que je vais exploser, mais je ne le montre pas). Plus le résultat est élevé, plus la propension à la suppression est fréquente. 
Tempérament colérique (alpha de Cronbach $=0,85$ ): Cette échelle de quatre items renvoie à la prédisposition plus ancrée à la colère. Un résultat élevé indique que l'adolescente a tendance à réagir promptement à la frustration et qu'elle s'emporte facilement (ex. : je me choque facilement, j'ai un caractère explosif).

Expression agressive de la colère (alpha de Cronbach $=0,87$ ): Cette échelle regroupe huit items qui évaluent à quelle fréquence des sentiments de colère sont exprimés par des comportements agressifs, que ce soit de façon verbale (ex. : quand je suis en colère, je dis des méchancetés) ou physique (ex. : quand je suis en colère, je fais des choses comme claquer des portes). Plus le résultat est élevé, plus l'expression de la colère au moyen de gestes agressifs est fréquente.

Régulation de la colère (alpha de Cronbach $=0,91$ ): Cette échelle est composée de huit items qui évaluent les mécanismes de régulation interne de la colère. Contrairement aux autres échelles, un résultat élevé trahit moins de difficultés, en ce sens que l'adolescente s'efforce de trouver des moyens pour apaiser ses sentiments de colère (ex. : quand je suis en colère, je prends une grande respiration et je relaxe; quand je suis en colère, je fais quelque chose de relaxant pour me calmer).

\section{Résultats}

Le profil des adolescentes des deux groupes lors de leur admission à l'unité d'hébergement est décrit au tableau 2. Ces résultats contrôlent statistiquement pour l'âge des adolescentes et sont rapportés sur une échelle de 1 à 4 . Dans l'ensemble, les adolescentes rapportent ressentir relativement peu de colère (moyenne $=2,05$; é.-t. = 0.92). Toutefois, elles affichent un tempérament plutôt colérique (moyenne $=2,68$; é.-t. $=0.90$ ). Elles expriment assez souvent leur colère par des gestes agressifs (moyenne $=2,63$; é.-t. $=0.75$ ) et ont une moindre tendance à supprimer leurs émotions de colère (moyenne $=2,46$; é.-t. $=0.53$ ). Leurs capacités à réguler leurs émotions de colère est plutôt faible (moyenne $=2,17$; é.-t. $=0,74$ ). Seule la régulation de la colère distinguait les adolescentes des deux groupes au pré-test, les adolescentes du groupe expérimental rapportant de plus faibles capacités à réguler leur colère $(F=4,28 ; p<0,05)$. Ce profil descriptif atteste des difficultés que présentent les adolescentes ciblées par la présente étude sur le plan de la régulation de la colère, difficultés qui se manifestent notamment par l'expression de comportements agressifs et par des réactions explosives. Notons que la colère ressentie, le tempérament colérique, la supression de la colère et l'expression agressive de la colère sont corrélées positivement les unes aux autres (les coefficients de corrélation variant entre 0,30 et 0,71$)$. La régulation de la colère est quant à elle corrélée négativement avec toutes ces variables (les coefficients de corrélation variant entre $-0,33$ et $-0,54)$, à l'exception de la suppression de la colère (corrélation non significative de -0,09). 
Tableau 2. Statistiques descriptives des dimensions de la colère évaluées à l'admission, selon le groupe

\begin{tabular}{|c|c|c|c|c|c|c|c|}
\hline & \multicolumn{2}{|c|}{ Échantillon global } & \multicolumn{2}{|c|}{ Groupe contrôle } & \multicolumn{2}{|c|}{$\begin{array}{c}\text { Groupe } \\
\text { expérimental }\end{array}$} & \multirow{2}{*}{$\begin{array}{c}\text { Test de } \\
\text { différences }\end{array}$} \\
\hline & Moyenne & é.-t. & Moyenne & é.-t. & Moyenne & é.-t. & \\
\hline $\begin{array}{l}\text { Colère } \\
\text { ressentie }\end{array}$ & 2,05 & 0,92 & 1,98 & 0,94 & 2,10 & 0,91 & $F=2,69$ \\
\hline $\begin{array}{l}\text { Suppression } \\
\text { de la colère }\end{array}$ & 2,46 & 0,53 & 2,46 & 0,48 & 2,47 & 0,56 & $F=0,21$ \\
\hline $\begin{array}{l}\text { Tempérament } \\
\text { colérique }\end{array}$ & 2,68 & 0,90 & 2,63 & 0,92 & 2,72 & 0,88 & $F=0,85$ \\
\hline $\begin{array}{l}\text { Expression } \\
\text { agressive }\end{array}$ & 2,63 & 0,75 & 2,59 & 0,81 & 2,66 & 0,70 & $F=0,02$ \\
\hline $\begin{array}{l}\text { Régulation } \\
\text { interne }\end{array}$ & 2,17 & 0,74 & 2,34 & 0,73 & 2,05 & 0,73 & $F=4,28$ * \\
\hline
\end{tabular}

Note : ${ }^{*}<0,05$

De façon à évaluer les effets du programme cognitif-comportemental sur ces dimensions de la colère, des analyses de trajectoires latentes ont été menées. Ces analyses sont en mesure d'établir comment évoluent les adolescentes de l'échantillon en ce qui concerne les dimensions ciblées et, surtout, d'évaluer à quel point l'exposition au programme cognitif-comportemental modifie le cours de la trajectoire des adolescentes sur ces dimensions'. L'effet de l'âge et de la continuité ou non du placement de chaque adolescente à chacun des temps de mesure a été contrôlé statistiquement.

Les analyses permettent d'abord de décrire l'évolution (la pente) de chacune des dimensions de la colère sur une période de 18 mois pour les adolescentes du groupe contrôle. Les résultats du tableau 3 indiquent que les adolescentes qui ne participent pas au programme cognitif-comportemental rapportent ressentir de moins en moins de colère au fil du temps $(\beta=-0,08 ; p<0,01)$. Elles ont aussi un peu moins tendance à supprimer leurs émotions de colère $(\beta=-0,04 ; p<0,01)$. Par contre, aucun changement significatif ne s'observe sur les autres dimensions de la colère. C'est donc dire que, prises globalement, les adolescentes du groupe contrôle demeurent avec un tempérament colérique $(\beta=-0,02 ; p>0,10)$, persistent à exprimer leur colère par des gestes agressifs $(\beta=-0,04 ; p>0,10)$ et n'améliorent pas leurs capacités à apaiser leurs sentiments de colère $(\beta=0,02 ; p>0,10)$.

1. Pour les quatre premières dimensions évaluées, les indices d'adéquation des modèles sont excellents. Le coefficient CFI est supérieur ou égal à 0,95 et le RMSEA est inférieur ou égal à 0,08 . Ces résultats permettent d'affirmer que les trajectoires estimées représentent adéquatement les données issues de notre échantillon (Hu et Bentler, 1999). Les indices d'adéquation sont légèrement plus faibles pour la variable « régulation de la colère ». 
Tableau 3. Évaluation des effets du programme sur les trajectoires de la colère sur une période de 18 mois, en contrôlant pour l'âge et pour le fait d'être hébergée ou non en centre de réadaptation à chacun des suivis

\begin{tabular}{|c|c|c|c|c|c|c|}
\hline & \multicolumn{4}{|c|}{$\begin{array}{l}\text { Coefficients d'adéquation } \\
\text { des modèles }\end{array}$} & \multicolumn{2}{|c|}{$\begin{array}{l}\text { Effets du programme } \\
\quad(0-18 \text { mois })\end{array}$} \\
\hline & $x^{2}$ & $\mathrm{p}$ & CFI & RMSEA & $\begin{array}{l}\text { Pente du } \\
\text { groupe contrôle }\end{array}$ & $\begin{array}{l}\text { Effet ajouté par } \\
\text { le groupe } \\
\text { expérimental }\end{array}$ \\
\hline Colère ressentie & 22,57 & 0,16 & 0,95 & 0,04 & 0,08 & 0,26 \\
\hline $\begin{array}{l}\text { Suppression de la } \\
\text { colère }\end{array}$ & 24,82 & 0,10 & 0,95 & 0,05 & 0,04 & 0,09 \\
\hline $\begin{array}{l}\text { Tempérament } \\
\text { colérique }\end{array}$ & 32,80 & 0,01 & 0,94 & 0,07 & 0,02 & 0,28 \\
\hline $\begin{array}{l}\text { Expression } \\
\text { agressive }\end{array}$ & 30,57 & 0,02 & 0,95 & 0,07 & 0,04 & 0,21 \\
\hline Régulation interne & 47,90 & 0,00 & 0,86 & 0,10 & 0,02 & 0,29 \\
\hline
\end{tabular}

Ensuite, la valeur ajoutée du programme cognitif-comportemental (l'appartenance au groupe expérimental) sur cette pente est estimée. Deux constats se dégagent des résultats. Premièrement, alors qu'une baisse significative de la colère ressentie par les adolescentes du groupe contrôle a été observée sur une période de 18 mois, cette baisse se trouve à être d'ampleur similaire chez les adolescentes du groupe expérimental $(\beta=-0,26 ; p>0.10)$. Il en est de même pour la baisse observée sur le plan de la suppression de la colère $(\beta=-0,09 ; p>0,10)$. Un tel résultat signifie que les adolescentes ayant été hébergées en centre de réadaptation s'améliorent sur ces aspects, qu'elles aient été exposées ou non au programme cognitif-comportemental. Deuxièmement, alors qu'aucun changement dans le temps n'a été observé pour le groupe contrôle au regard du tempérament colérique, de l'expression agressive de la colère et de la régulation de la colère, toutes ces dimensions s'améliorent dans le temps pour les adolescentes du groupe expérimental (respectivement $\beta=-0,28, p<0.05 ; \beta=-0,21, p<0.05 ; \beta=0,29, p<0.01$ ). Ces résultats signifient que ces difficultés demeurent constantes et persistantes au fil du temps chez les adolescentes du groupe contrôle, alors qu'elles s'atténuent significativement chez celles qui ont été exposées au programme cognitif-comportemental. Les figures 1 à 5 illustrent les effets du programme en traçant les trajectoires respectives des adolescentes des groupes contrôle et expérimental ${ }^{2}$.

2. Considérant que la variance observée pour chacune des dimensions de la colère est relativement restreinte, les graphiques rapportent les résultats sur une échelle dont le minimum et le maximum renvoient aux valeurs se situant à plus ou moins un écart-type de la moyenne. 


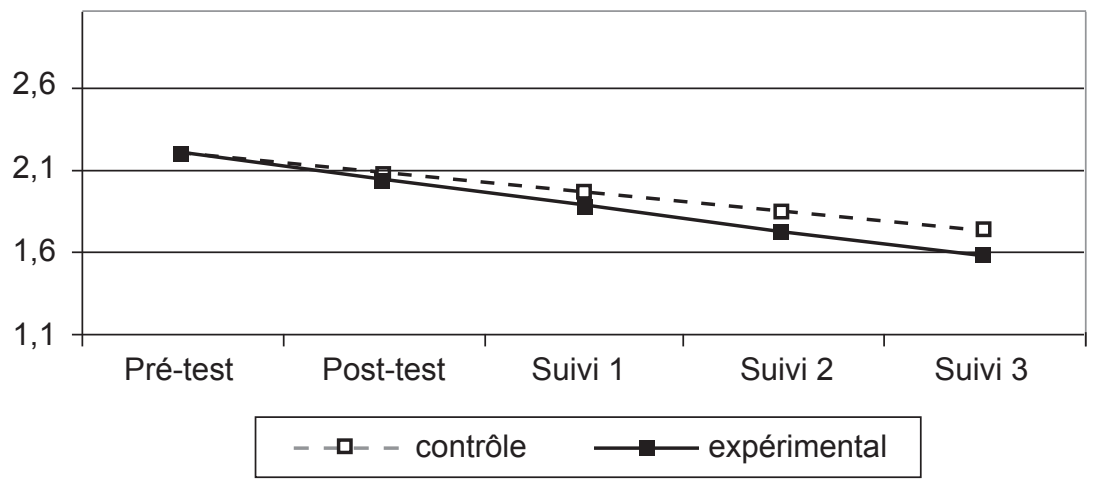

Figure 1. Effets du programme sur la colère ressentie

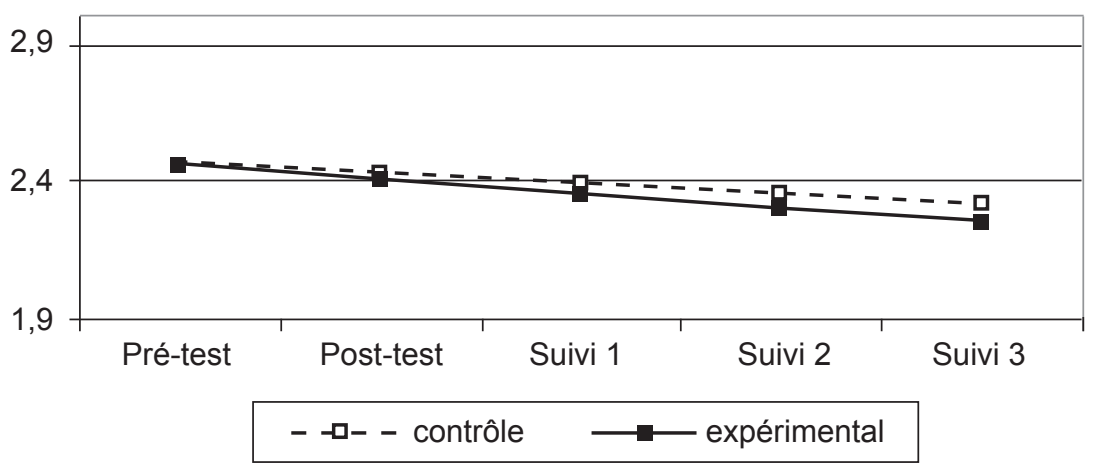

Figure 2. Effets du programme sur la suppression de la colère

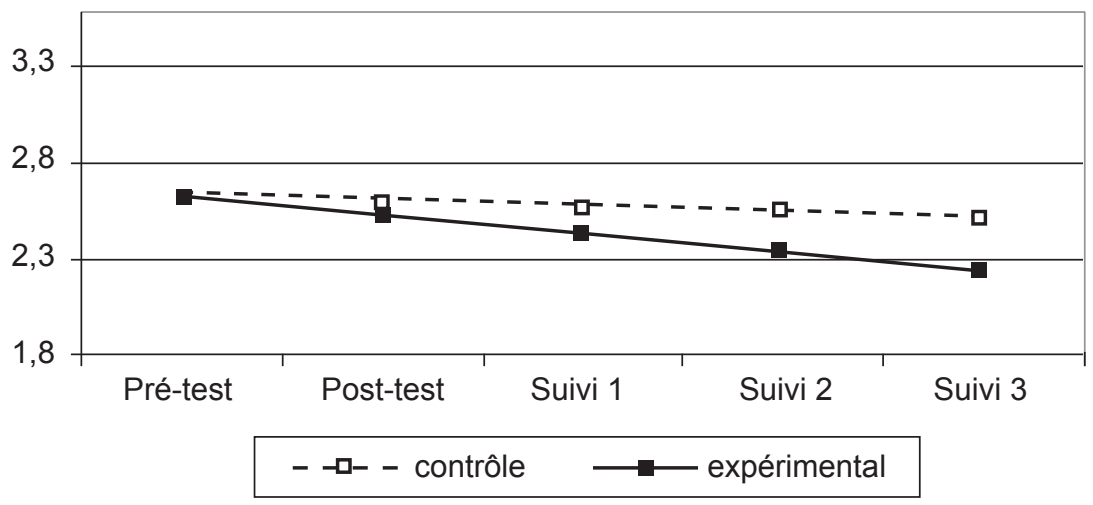

Figure 3. Effets du programme sur le tempérament colérique 


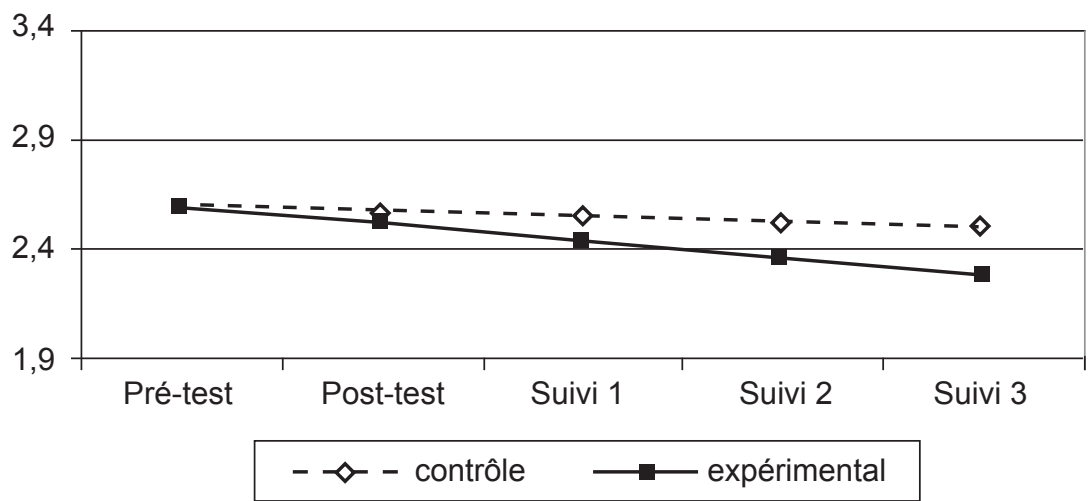

Figure 4. Effets du programme sur l'expression agressive de la colère

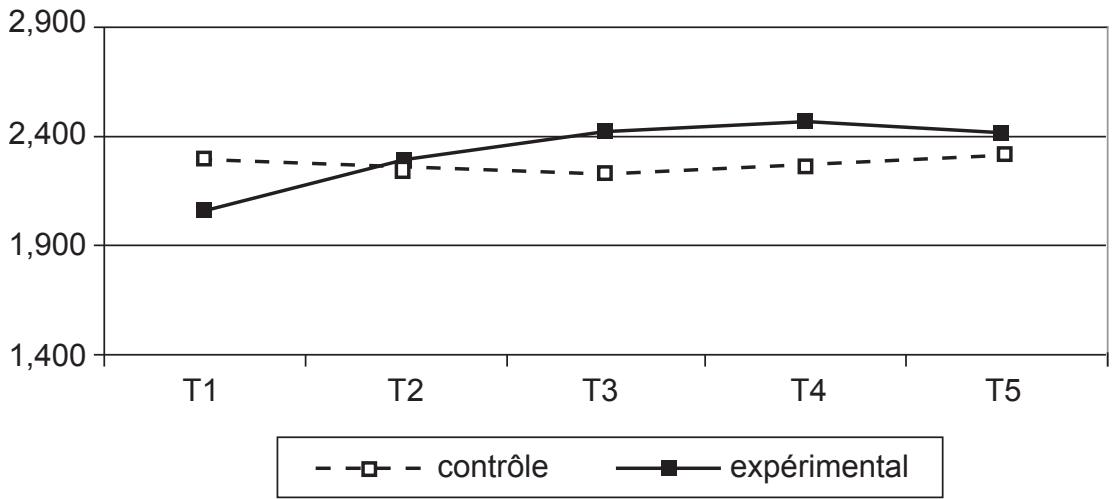

Figure 5. Effets du programme sur la régulation de la colère

De façon à mieux qualifier les effets du programme cognitif-comportemental, la taille de ces effets est précisée au moyen du coefficient $d$ de Cohen (1988) ${ }^{3}$. Ce coefficient est précisé pour chacun des temps de mesure, soit 3 mois, 6 mois, 12 mois et 18 mois après le début du programme. Le coefficient $d$ de Cohen renvoie à des repères standardisés largement utilisés dans le domaine de l'évaluation de programmes. De plus, des seuils cliniques y sont associés, ce qui permet de mieux saisir la portée des résultats dans un contexte d'intervention (Wolf, 1986). Plus précisément, il est postulé que des coefficients de 0,25 à 0,49 indiquent une amorce de changement chez les individus, et que des coefficients de 0,50 et plus reflètent un changement cliniquement significatif. La figure 6 indique que les deux variables pour lesquelles il a été observé que le programme cognitif-comportemental

3. La taille des effets a été calculée en référence aux données observées au temps 1 , ceci pour chaque temps de mesure subséquent (tempsx où $x$ varie de 2 à 5 ) à l'aide de cette formule : $\mu_{1}-\mu_{x} \mid / \sqrt{ }\left(\left(S_{\mu 1}+S_{\mu x}\right) / 2\right)$. 
n'apporte pas de valeur ajoutée (colère ressentie et suppression de la colère) ont une taille de l'effet qui se situe sous la barre du 0,25 , ce qui réitère l'absence d'effet du programme sur ces dimensions. Une amorce de changement s'observe pour les autres dimensions de la colère. Quoique les effets soient plutôt modestes, ils tendent à se maintenir dans le temps, ceci même si la plupart des adolescentes ne sont plus exposées au programme après une période de 6 mois. Les changements les plus significatifs sur le plan clinique se situent dans la capacité des adolescentes à mieux réguler leur colère. Les résultats indiquent même que cet effet s'accentue au fil du temps pour franchir le seuil du $d=0,50$ au bout de 18 mois.

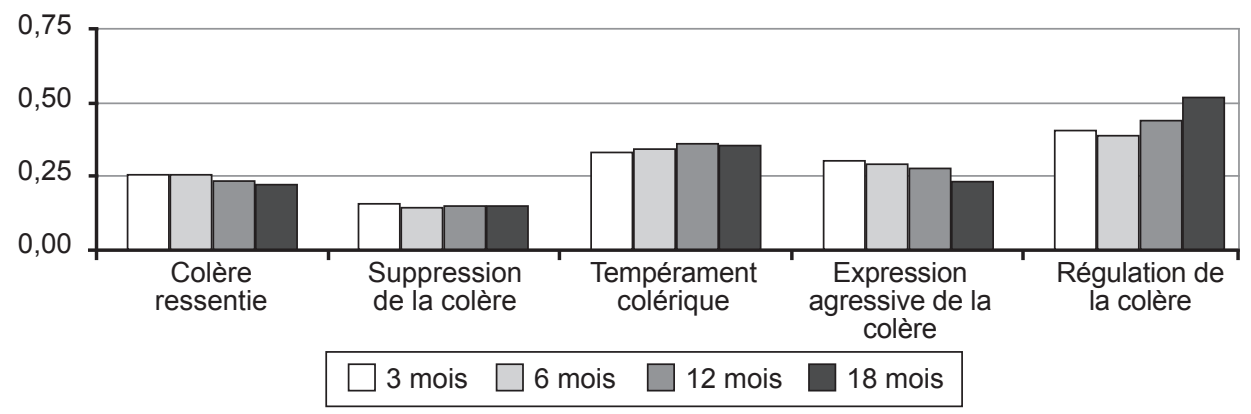

Figure 6. Taille des effets du programme cognitif-comportemental à chacun des temps de mesure sur les dimensions de la colère

\section{Discussion}

Depuis quelques années, l'adéquation des services et des programmes destinés aux adolescentes prises en charge par la justice suscite beaucoup de questionnements (Hubbard \& Matthews, 2008). La principale question qui est soulevée réfère à la nécessité de développer des programmes qui soient spécifiques ou non à cette clientèle (Belknap \& Holsinger, 1998; Bloom et al., 2002; KempfLeonard \& Sample, 2000; Lanctôt, 2006). Une réponse à ce propos nécessite la conduite de recherches évaluatives permettant d'identifier des pratiques probantes pour cette clientèle bien précise (Lanctôt, 2010). Or, la littérature fait état d'une quasiabsence de recherches évaluatives menées auprès d'échantillons d'adolescentes qui présentent des difficultés de comportement (Hipwell \& Loeber, 2006; Zahn et al., 2009). Dans ce contexte, certains se montrent réticents à appliquer des programmes de type cognitif-comportemental à des adolescentes en difficulté, sous prétexte que ces programmes sont qualifiés de probants pour les délinquants masculins.

La présente étude s'inscrit dans la volonté de combler ce vide dans la littérature. Elle s'est attardée à évaluer les effets d'un programme d'intervention de type cognitif-comportemental sur la régulation et l'expression de la colère chez des adolescentes hébergées en Centres jeunesse. En effet, les programmes de nature cognitive-comportementale ciblent des déficits liés à la régulation des émotions et leurs effets sur les problèmes reliés à la colère et sur les conduites agressives semblent prometteurs (voir notamment les méta-analyses de Beck 
\& Fernandez, 1998; Blake \& Hamrin, 2007; Sukhodolsky, Kassinove \& Gorman, 2004). Par ailleurs, l'étude de Lanctôt (2006) suggèrent que les adolescentes et les adolescentes hébergées en Centre jeunesse classent elles-mêmes la participation à des activités visant la régulation de la colère parmi leurs besoins d'intervention prioritaires.

L'évaluation des effets du programme sur la capacité des adolescentes à réguler leur colère et à l'exprimer de façon prosociale a été menée au moyen d'un devis de recherche quasi-expérimental sur une période de 18 mois. Les résultats indiquent que le programme apporte une certaine plus-value à la démarche de réadaptation. Les adolescentes placées dans les unités d'hébergement où le programme cognitif-comportemental était appliqué se sont améliorées à plusieurs égards au fil du temps, en comparaison aux adolescentes du groupe contrôle. Ces améliorations se traduisent par une diminution du tempérament colérique et de la fréquence des manifestations de colère ainsi que par une meilleure capacité à réguler les émotions de colère. Ces résultats sont forts prometteurs, car les effets bénéfiques observés se situent au cœur des cibles d'intervention visées par le programme cognitif-comportemental.

Par ailleurs, le programme ne semble pas avoir d'effets spécifiques au plan de la colère ressentie et de la suppression de la colère. Ces aspects s'atténuent tranquillement au fil du temps chez les adolescentes ayant été placées en centre de réadaptation, que ces dernières aient été exposées ou non au programme cognitif-comportemental. Ces observations sont congruentes avec les objectifs du programme cognitif-comportemental. En effet, ce dernier ne vise surtout pas à faire en sorte que les adolescentes se sentent mois en colère ou qu'elles n'extériorisent plus leurs émotions. Le programme vise plutôt à ce que les adolescentes acquièrent une meilleure capacité de régulation de leur colère et apprennent à l'exprimer d'une façon prosociale. Les résultats se positionnent donc en faveur de l'application d'un programme de type cognitif-comportemental à des adolescentes hébergées en Centre jeunesse.

Plusieurs autres pistes d'analyse devront être approfondies. II importera de vérifier si les effets s'étendent à d'autres dimensions, comme les cognitions et les émotions qui se rattachent à la détresse émotive. II importera aussi de vérifier si ces effets se généralisent à d'autres contextes de vie (comme les relations avec les parents et avec les enseignants) et s'ils se maintiennent à plus long terme. Enfin, la présence d'effets de médiation ou de modération devra être évaluée. D'une part, les liens qui s'établissent entre les pensées, les émotions et les comportements devront être précisés. D'autre part, l'effet modérateur de certains facteurs (comme les antécédents d'agression sexuelle, les problèmes de toxicomanie ou de santé mentale et même la qualité de l'alliance thérapeutique) devra être évalué, comme le suggèrent Hipwell \& Loeber (2006) ainsi que Hubbard \& Matthews (2008). Il importe aussi de relever que notre étude n'est pas exempte de limites. Notamment, la mesure de la suppression de la colère présente une cohérence interne relativement faible. Aussi, les analyses n'ont pas contrôlées statistiquement pour le degré d'expostion au programme. Cette piste devra assurément être approfondie, d'autant plus que des lacunes ont été notées quant au dosage des différentes composantes du programme. II est fort probable qu'une exposition plus soutenue au programme 
aurait donné lieu à des effets plus marqués. Néanmoins, notre étude a le mérite d'évaluer un programme non pas dans un contexte idéal de laboratoire, mais bel et bien dans un contexte réel de pratique, aussi imparfait soit-il.

Enfin, malgré que l'analyse que nous avons faite des résultats dans le cadre de cet article se limite aux effets du programme sur des dimensions reliées à la colère, notre étude possède des forces qui doivent être mentionnées. Cette étude est en effet l'une des rares dans le domaine de la délinquance féminine à évaluer un programme d'intervention au moyen d'un devis quasi-expérimental, à recourir à un échantillon relativement grand et à rapporter la taille des effets observés. Pour toutes ces raisons, les résultats présentés peuvent contribuer à identifier les meilleures pratiques à privilégier auprès des adolescentes qui présentent des difficultés de comportement. Bien que nos résultats devront être répliqués, notre étude suggère néanmoins que l'application d'un programme cognitif-comportemental réduit de façon significative des difficultés au regard du tempérament colérique, de l'expression agressive de la colère et de la régulation de la colère chez les adolescentes hébergées en Centre jeunesse. En ce sens, alors que certains prônent le développement de programmes qui soient spécifiques à la clientèle féminine du système de justice pour mineurs, nos résultats appuient d'abord et avant tout le développement de programmes basés sur des données probantes.

\section{Références}

Ayotte, M.-H., Lanctôt, N., Lemieux, A., \& Giroux, É. (2009). Évaluation des effets du programme cognitif-comportemental sur la délinquance des adolescentes et sur ses facteurs associés auprès d'un groupe contrôle (CJL) et d'un groupe expérimental (CJM-IU). Rapport de recherche déposé au Conseil national de la prévention du crime, Canada : Ottawa.

Barnett, G. D. (2012). Gender-responsive programming: A qualitative exploration of women's experiences of a genderneutral cognitive skills programme. Psychology, Crime, \& Law, 18 (2), 155176.

Beck, R., \& Fernandez, E. (1998). CognitiveBehavioral Therapy in the Treatment of Anger: A Meta-Analysis. Cognitive Therapy and Research, 22 (1), 63-74.

Bélanger, A., \& Ouimet, M. (2010). L'évolution de la délinquance féminine. Une analyse des données officielles canadiennes entre 1974 et 2003. Revue internationale de criminologie et de police technique et scientifique, 63 (1), 66-85.
Belknap, J., \& Holsinger, K. (1998). An overview of delinquent girls: How theory and practice have failed and the need for innovative changes. Dans $R$. Zaplin (dir.), Female offenders Critical perspectives and effective intervention (pp. 31-59). Gaithersburg, MD: Aspen Publishers.

Bjorkqvist, K., Lagerspetz, K. M. J., \& Osterman, K. (1992). The Direct and Indirect Aggression Scales. Vasa, Finlad: Abo Akademi University, Department of Social Sciences.

Bjorkqvist, K., \& Niemela, P. (1992). Of mice and women: Aspects of female aggression. San Diego: Academic Press.

Blake, C. S., \& Hamrin, V. (2007). Current Approaches to the Assessment and Management of Anger and Aggression in Youth: A Review. Journal of Child and Adolescent Psychiatric Nursing, 20 (4), 209-221.

Bloom, B., Owen, B., Deschenes, E., \& Rosenbaum, J. (2002). Moving toward 
justice for female juvenile offenders in the new millennium. Journal of Contemporary Criminal Justice, 18 (1), 37-56.

Cohen J. (1988). Statistical Power Analysis for the Behavioral Sciences (2nd ed.), Hillsdale, NJ : Erlbaum.

Cole, P. M., Zahn-Waxler, C., Fox, N. A., Usher, B. A., \& Welsh, J. D. (1996). Individual differences in emotion regulation and behavior problems in preschool children. Journal of Abnormal Psychology, 105, 518-529.

Cole, P. M., Michel, M. K., \& Teti, L. O. (1994). The development of emotion regulation and dysregulation: A clinical perspective. Monographs of the Society for Research in Child Development, 59 (2-3), The Development of Emotion Regulation: Biological and Behavioral Considerations, 73-100.

Conway, A. M. (2005). Girls, Aggression, and Emotion Regulation. American Journal of Orthopsychiatry, 75 (2), 334-339.

Cornell, D. G., Peterson, C. S., \& Richards, H. (1999). Anger as a predictor of aggression among incarcerated adolescents. Journal of Consulting and Clinical Psychology, 67 (1), , 108-115.

Crick, N.R., Bigbee, M.A., \& Howes, C. (1996). Children's normative beliefs about aggression: How do I hurt thee? Let me count the ways. Child development, 67 (3), 1003-1014.

Crick, N. R., \& Grotpeter, J. K. (1995). Relational aggression, gender, and social-psychological adjustment. Child Development, 66, 710-722.

Crick, N.R., \& Dodge, K.A. (1994). A review and reformation of social information processing mechanisms in children's social adjustment. Psychological Bulletin, 115, 74-101.

Cummings, A.L., \& Leschied, A.W. (2002). Research and treatment for aggression with adolescent girls. Lewiston, NY: The Edwin Mellen Press.

Fabes, R. A., Eisenberg, N., Jones, S., Smith, M., Guthrie, I., Poulin, R., Shepard, S., \& Friedman, J. (1999). Regulation, emotionality, and preschoolers' socially competent peer interactions. Child Development, 70 (2), 432-442.

Fives, C., Kong, G., Fuller, J., \& DiGiuseppe, R. (2011). Anger, aggression, and irrational beliefs in adolescents. Cognitive Therapy and Research, 35 (3), 199-208.

Foley, A. (2008). The current state of genderspecific delinquency programming. Journal of Criminal Justice, 36 (3), 262269.

Gobeil, A. (2011). Rumination de la tristesse et de la colère et les comportements agressifs chez les adolescentes en difficulté. Mémoire de maîtrise. Université de Sherbrooke, Sherbrooke.

Hipwell, A. E., \& Loeber, R. (2006). Do we know which interventions are effective for disruptive and delinquent girls? Clinical Child and Family Review, 9 (34), 221-255.

Hu, L., \& Bentler, P. M. (1999). Cutoff criteria for fit indexes in covariance structure analysis: Conventional criteria versus new alternatives. Structural Equation Modeling, 6 (1), 1-55.

Hubbard, D. J., \& Matthews, B. (2008). Reconciling the differences between the "gender-responsive» and the "what works» literatures to improve services for girls. Crime \& Delinquency, 54 (2), 225-258.

Keenan, K. (2000). Emotion dysregulation as a risk factor for child psychopathology. Clinical Psychology: Science and Practice, 7, $418-434$.

Kempf-Leonard, K., \& Sample, L. L. (2000). Disparity based on sex: Is genderspecific treatment warranted? Justice Quarterly, 17 (1), 89-128.

Kendall, K., \& Pollack, S. (2003). Cognitive behavioralism in women's prisons: A critical analysis of therapeutic assumptions and practices, Dans B. E. Bloom (dir.), Gendered justice: Addressing female offenders (pp. 4568), Durham, NC: Carolina Academic Press. 
Lanctôt, N. (2006). Doit-on définir des programmes d'intervention spécifiques pour réduire la déviance des adolescentes?. Dans P. Verlaan, \& M. Déry (dir.), Les conduites antisociales chez les filles: Comprendre pour mieux agir (pp. 415-439). Ste-Foy, QC : Presses de l'Université du Québec.

Lanctôt, N. (2010). La délinquance féminine : un caractère spécifique à nuancer. Dans M. Le Blanc, \& M. Cusson (dir.), Traité de criminologique empirique, $4 \mathrm{e}$ édition. Montréal : Les Presses de l'Université de Montréal.

Landenberger, N.A., \& Lipsey, M.W. (2005). The positive effects of cognitivebehavioral programs for offenders: A meta-analysis of factors associated with effective treatment. Journal of Experimental Criminology, 1, 451-476.

Latimer, J., Dowden, C., Morton-Bourgon, K.E., Edgar, J., \& Bania, M. (2003). Treating youth in conflict with the law: A new meta-analysis, Research and Statistics Division, Department of Justice, Canada.

Lauritsen, J. L., Heimer, K., \& Lynch, J. P. (2009). Trends in the gender gap in violent offending: New evidence from The National Crime Victimization Survey. Criminology, 47, 361-399.

Le Blanc, M., Dionne, J., Proulx, J., Grégoire, J. C., \& Trudeau-Le Blanc, P. (1998). Intervenir autrement : Un modèle différentiel pour les adolescents en difficulté. Montréal : Les Presses de I'Université de Montréal.

Lipsey, M. W. (1990). Juvenile delinquency treatment: A Meta-analytic inquiry into the variability of effects. New York, Russell Sage Foundation.

Loeber, R., \& Hay, D. (1997). Key issues in the development of aggression and violence from childhood to early adulthood. Annual Review of Psychology, 48, 371 $-410$

Lösel, F., \& Beelmann, A. (2006). Child social skills training. Dans B.C. Welsh, \& D.P. Farrington (dir.), Preventing crime: What works for children, offenders, victims and places (pp.33-54). Dordrecht, Netherlands: Springer.

Marsee, M., \& Frick, P. (2007). Exploring the Cognitive and Emotional Correlates to Proactive and Reactive Aggression in a Sample of Detained Girls. Journal of Abnormal Child Psychology, 35 (6), 969981.

McCart, M. R., Priester, P. E., Davies, W. H., \& Azen, R. (2006). Differential effectiveness of behavioral parenttraining and cognitive-behavioral therapy for antisocial youth: A meta-analysis. Journal of Abnormal Child Psychology, 34 (4), 525-541.

Miller, N., Pedersen, W. C., Earleywine, M., \& Pollock, V. E. (2003).A theoretical Model of triggered displaced aggression. Personal and Social Psychology Review, 7, 75-97.

Nichols, T. R., Graber, J. A., Brooks-Gunn, J., \& Botvin, G. J. (2006). Sex differences in overt aggression and delinquency among urban minority middle school students. Journal of Applied Developmental Psychology, 27 (1), 7891.

Nolen-Hoeksema, S. (2004). The response styles theory. Dans C. Papageorgiou, \& A. Wells (dir.), Depressive rumination: Nature, theory, and treatment (pp. 107124). New York: Wiley.

Odgers, C., \& Moretti, M. (2002). Aggressive and antisocial girls: Research update and challenges. International Journal of Forensic Mental Health, 1 (2), 103-119.

Orobio de Castro, B., Merk, W., Koops, W., Veerman, J.W., \& Bosch, J.D. (2005). Emotions in social information processing and their relations with reactive and proactive aggression in referred aggressive boys. Journal of Clinical Child and Adolescent Psychology, 34, 105-116.

Peled, M., \& Moretti, M. (2010). Ruminating on Rumination: are Rumination on Anger and Sadness Differentially Related to Aggression and Depressed Mood?. Journal of Psychopathology and Behavioral Assessment, 32 (1), 108117. 
Peled, M., \& Moretti, M. (2007). Rumination on anger and sadness in adolescence: Fueling of fury and deepening of despair. Journal of Clinical Child and Adolescent Psychology, 36, 66-75.

Repper, K. K. (2006). The relationship between anger rumination and aggression in childhood. Thèse de doctorat. Florida State University, Tallahassee.

Silk, J. S., Steinberg, L., \& Morris, A. S. (2003). Adolescents' Emotion Regulation in Daily Life: Links to Depressive Symptoms and Problem Behavior. Child Development, 74 (6), 1869-1880.

Simmons, R. (2002). Odd girl out: The hidden culture of aggression in girls. New York: Harcourt.

Spielberger, C. D. (1999). State-Trait Anger Expression Inventory-2 (STAXI-2). Odessa, FI: Psychological Assessment Resource Inc.

Sukhodolsky, D. G., Golub, A., \& Cromwell, E. N. (2001). Development and validation of the anger rumination scale. Personality and Individual Differences, 31(5), 689-700.
Sukhodolsky, D. G., Kassinove, H., \& Gorman, B. S. (2004). Cognitivebehavioral therapy for anger in children and adolescents: A meta-analysis. Aggression and Violent Behavior, 9 (3): 247-269.

Verlaan, P., Besnard, T., Déry, M., Toupin, J., \& Pauzé, R.(2009). Agresser sans frapper : un regard sur les conduites d'agression indirecte. Dans B.H. Schneider, S. Normand, M. Alles-Jardel, M.A. Provost, \& G.M. Tarabulsy (dir.), Conduites agressives chez l'enfant. Perspectives développementales et psychosociales (p. 37-56). Québec : PUQ.

Zahn, M. A., Day, J. C., Mihalic, S. F., \& Tichavsky, L. (2009). Determining what works for girls in the juvenile justice system: A summary of evaluation evidence. Crime and Delinquency, 55 (2), 266-293. 